\title{
Délit politique et terrorisme en Belgique : du noble
} au vil

\section{Martin Moucheron}

\section{(2) OpenEdition \\ 1 Journals}

Édition électronique

URL : http://journals.openedition.org/conflits/2038

DOI : 10.4000/conflits.2038

ISSN : $1777-5345$

Éditeur :

CCLS - Centre d'études sur les conflits lilberté et sécurité, L'Harmattan

\section{Édition imprimée}

Date de publication : 1 mars 2006

Pagination : 77-100

ISBN : 2-296-00622-1

ISSN : 1157-996X

Référence électronique

Martin Moucheron, « Délit politique et terrorisme en Belgique : du noble au vil », Cultures \& Conflits [En ligne], 61 | printemps 2006, mis en ligne le 17 mai 2006, consulté le 30 mars 2021. URL : http://

journals.openedition.org/conflits/2038; DOI : https://doi.org/10.4000/conflits.2038

Ce document a été généré automatiquement le 30 mars 2021.

Creative Commons License 


\title{
Délit politique et terrorisme en Belgique : du noble au vil
}

\author{
Martin Moucheron
}

1 A la différence de la France, qui disposait déjà d'une incrimination particulière du terrorisme, l'inscription d'infractions terroristes spécifiques dans le Code pénal, en date du 19 décembre 2003 et à la suite de la décision-cadre de l'Union européenne du 13 juin 2002, constitue une innovation sans précédent en Belgique. En effet, les actes les plus consensuellement qualifiés de "terroristes " par le grand public - et notamment ceux qui ont secoué le Royaume dans les années 1980 à travers les attentats à la bombe commis par les Cellules communistes combattantes (CCC $)^{1}$ - étaient jusqu'alors soumis au régime du droit commun, sans que leur qualification pénale ne soit influencée par la dimension proprement politique de ces actions. Ce régime pénal « ordinaire » appliqué au terrorisme a connu une dernière application judiciaire en juin 2004, où la peine de dix ans d'emprisonnement infligée en 2003 à Nizar Trabelsi, qui a avoué avoir projeté en septembre 2001 un attentat à l'explosif contre une base militaire belge en lien avec Al-Qaïda, a été confirmée en appel sur base de la législation existante - et principalement l'article 322 du Code pénal relatif à l'association formée dans le but d'attenter aux personnes ou aux propriétés ${ }^{2}$. A contrario, l'existence d'un régime particulier aux délits commis avec une intention politique a été consacrée aux premiers jours de l'Etat belge à travers les dispositions relatives au délit politique, garantissant à l'auteur d'un tel délit un statut quelque peu privilégié, tant au niveau des poursuites et $\mathrm{du}$ jugement que de l'exécution de la peine. Même si la portée effective de ces dispositions a progressivement été réduite par le truchement de la jurisprudence jusqu'à les rendre quasi inapplicables, le principe même d'un traitement de faveur pénal envers les infractions mues par des objectifs politiques n'était pas remis en question. Cependant, l'inscription en droit belge d'infractions terroristes dont la définition repose sur un élément intentionnel particulier, assimilable à un objectif politique sensu lato marque un revirement dans la tradition de mansuétude à l'égard du délit politique : les infractions terroristes sont en effet sanctionnées plus sévèrement en raison même de cette intention particulière, ce qui indique une orientation diamétralement opposée à celle introduite par l'idéologie libérale classique qui a guidé 
la plume des rédacteurs des textes fondateurs de l'Etat belge de 1830, Constitution en tête. Cette inversion de tendance, confirmée par de multiples sources empiriques et dépassant les frontières du Royaume, peut être interprétée comme indicative d'une évolution récente de la gouvernementalité ${ }^{3}$, et plus particulièrement révélatrice d'un changement dans la perception du mode contemporain de gouvernementalité comme forme la plus aboutie, absolue, de la société démocratique, qu'il convient dès lors de défendre contre toute velléité de changement.

Le délit politique en Belgique : la longue histoire d'un régime de faveur

2 Unie depuis 1815 au royaume des Pays-Bas des Princes d'Orange, la Belgique acquiert son indépendance par la force en septembre 1830, lors d'un soulèvement populaire qui rejette les Hollandais vers le Nord. Avec la bénédiction tacite des cinq grandes puissances de l'époque (Angleterre, France, Autriche, Prusse, Russie), elle s'établit en tant que royaume de Belgique et se dote, en février 1831, d'une Constitution originale mais inspirée des constitutions françaises de 1791, 1814 et 1830, de la Constitution néerlandaise de 1814 et du droit constitutionnel anglais. A une époque où s'est imposée en philosophie politique la doctrine libérale classique, les principes fondamentaux du jeune Etat, qui a conquis de haute lutte son autonomie par les armes contre la volonté de Guillaume $\mathrm{I}^{\mathrm{er}}$, son souverain légitime, consacrent une catégorie particulière de délit : le délit politique.

Le délit politique originel : un régime de faveur

3 La Constitution de 1830 établit d'emblée une distinction claire : de plusieurs points de vue, il convient de réserver un traitement particulier aux délits politiques. Si le délit politique a bénéficié en Belgique d'une attention particulière, c'est en raison des circonstances historiques de la naissance de l'Etat belge. En effet, nombre d'acteurs de la fondation de la Belgique (membres constituants, premiers élus, etc.) se sont opposés à la tutelle hollandaise pour arracher l'indépendance, et ont été poursuivis par la justice des Pays-Bas en raison de cet engagement ${ }^{4}$. Mais cette discrimination de la contestation politique s'inspire également des courants de pensée de l'époque, comme l'écrivent F. Tulkens et M. van de Kerchove: "La Révolution française et le mouvement libéral du XIXe siècle transformèrent le "crime d'Etat" en "délit politique" dont l'immoralité, pour le droit pénal classique, n'apparaît ni aussi claire ni aussi immuable que celle des crimes privés $^{5}$ ». Dès lors, les textes fondateurs et initiaux de la Belgique reflètent cette préoccupation : « en droit positif, la Constitution de 1831 - [...] - le Code pénal de 1867 ainsi que certaines lois particulières reconnaissent le délit politique en lui accordant un régime répressif particulier $[. . .]^{6} \gg$ et il s'agit en l'espèce d'un traitement plus favorable.

4 En effet, comme l'exposent ces auteurs, les délits politiques ont joui d'un statut juridique privilégié, et ce à plusieurs égards: «traditionnellement, le régime répressif applicable aux délits politiques est considéré comme étant un régime de faveur ${ }^{7}$ ». Compétence de la Cour d'assises même en matière de délit (qui laisse le peuple seul juge pour les délits politiques), régimes pénal, procédural et pénitentiaire assouplis, principe de nonextradition, autant de particularités associées au délit et au crime politique, même si, tout au long $\mathrm{du} \mathrm{XIX}^{\mathrm{e}}$ siècle, ce traitement de faveur a été progressivement restreint comme nous le verrons plus loin. Sur le fond, il s'agissait de consacrer juridiquement le principe selon lequel un acte illégal accompli avec un dessein politique, s'il reste condamnable en vertu des moyens, manifeste une certaine " grandeur d'âme » dans le chef de son auteur en vertu de la fin poursuivie qui impose d'être distinguée des motivations les plus basses des délits de droit commun. 
5 En quelque sorte, le rousseauisme ambiant imposait de singulariser la noblesse des infractions motivées par des objectifs de changement politique : de tels délinquants, malgré l'indispensable répression que suscitent leurs actes qui contreviennent aux règles du vivre-ensemble, expriment pourtant de hautes qualités humaines et leur pleine appartenance à la société en ce qu'ils se saisissent, par idéalisme et au mépris des sanctions encourues, de la res publica. En cela, ils se soucient plus du bien commun que de leur intérêt propre, et sont bien plus proches du citoyen, sujet politique libre, que ne l'est l'auteur d'infractions poussé par le but de lucre ou la vengeance. De plus, l'idéal libéral proprement dit impose la liberté la plus large, et particulièrement dans une Belgique bourgeoise dont le clivage politique entre catholiques et libéraux a repris vigueur une fois vaincu l'ennemi commun - elle est d'ailleurs constitutionnellement consacrée en matière d'enseignement, de presse et d'association, notamment.

Plus fondamentalement, on peut en conclure que le pouvoir constituant de 1830, attaché au plus haut point à ces principes libéraux, ne connaissait que trop bien l'incertitude liée à l'évolution de la société et à la praxis de l'art de gouverner, devant sa propre légitimité à une révolution qui a bouleversé les cadres politiques établis. Pour cette raison, il a conçu un régime particulier octroyant un statut juridique plus favorable à l'auteur d'un délit politique. Pour l'assemblée constituante, l'élément intentionnel du délit politique témoigne dans le chef de son auteur d'un souci pour le bien collectif contrastant avec les basses préoccupations individualistes des délits de droit commun, méritant dès lors un traitement pénal plus modéré. Cependant, en établissant ce régime de faveur, le pouvoir constituant reconnaissait également, de façon implicite, le caractère contingent de la forme particulière du gouvernement qu'il exerçait, au regard des principes absolus qu'il visait et défendait.

7 Cependant, ni la Constitution de 1830, ni le Code pénal de 1867, ni les lois particulières n'offrent de définition du délit politique, laissant à la jurisprudence le soin d'en établir les contours, et de suivre de façon plus adaptative l'évolution de la société. Or, c'est par ce biais que la notion de délit politique sera progressivement restreinte au fil du xIX et $\mathrm{du} \mathrm{xx}^{\mathrm{e}}$ siècle jusqu'à rendre son application effective... quasi-inapplicable, sans pour autant que le principe même d'un statut privilégié pour celui-ci ne soit légalement ou constitutionnellement remis en cause.

De la patrie révolutionnaire à l'Etat conservateur : la restriction du délit politique

8 Le souffle libéral et révolutionnaire qui a porté le mouvement d'indépendance de la Belgique n'a qu'un temps: une fois pérennisés les acquis de 1830, les gouvernements successifs se montreront de plus en plus soucieux de conjuguer ces principes libéraux avec la stabilité des formes politiques, économiques et sociales de l'Etat. Dès lors, l'extension de la notion de délit politique, apportée par la jurisprudence, se verra progressivement restreinte, sinon dans les premières années de l'existence du Royaume, tout du moins à partir de la seconde moitié du XIX siècle. En effet, l'émergence de revendications sociales radicales et de la représentation d'une société de classes fait craindre des bouleversements de l'ordre établi, dont la Commune de Paris de 1871 constitue la démonstration ${ }^{8}$; ainsi, la jurisprudence inaugure à cette époque le long mouvement de durcissement progressif dans les conditions effectives de la qualification du délit politique. Tel que défini aujourd'hui par la Cour de cassation, il est nécessaire, pour accorder une telle qualification à un délit, de constater l'existence d'un élément matériel et d'un élément intentionnel particuliers - également qualifiés $d^{\prime}$ « objectif» et de « subjectif " par certains auteurs ${ }^{9}$ : 
9 - l'élément matériel de l'infraction est l'atteinte directe à l'ordre politique de l'Etat, qu'il soit « intérieur » - incluant « tout ce qui se rattache aux pouvoirs politiques établis par la Constitution: forme de l'Etat, autorité et prérogatives constitutionnelles du Roi, formation et fonctionnement des assemblées législatives, communautaires ou régionales, exercice du pouvoir par les ministres, droits politiques des citoyens, etc.» - ou " extérieur» - comprenant "l'indépendance de la nation, l'intégrité du territoire, les relations entre Etats ${ }^{10}$ ». On distingue d'une part le délit politique pur, qui par nature porte seulement atteinte à l'ordre politique (à l'exemple des infractions en matière électorale ${ }^{11}$ ), et d'autre part le délit politique complexe ou mixte, qui est une infraction de droit commun qui doit son caractère politique aux circonstances de sa commission ${ }^{12}$.

10 - l'élément intentionnel de l'infraction est l'objectif visé par l'auteur : celui-ci doit avoir agi dans un but politique. Dès lors, par exemple, les infractions commises avec un but de lucre ne peuvent être qualifiées de politiques, et ce même si l'élément matériel est présent.

11 De plus, sous l'influence de l'évolution du champ des idées en matière de fondement de l'intervention pénale, la conception même du délit politique comme délit "noble » devant bénéficier d'un traitement de faveur s'effrite quelque peu au fil des années, et ce, dès le début du siècle dernier ${ }^{13}$. Malgré son opposition au courant pénal classique, l'école positiviste italienne soutiendra encore au début $d u x^{e}$ siècle que l'auteur du délit politique n'est guère différent de l'homme normal, et qu'il peut même être vu comme un visionnaire ou un génie en avance sur son temps ${ }^{14}$. Néanmoins, l'immédiat après-guerre voit grandir l'influence du courant de la défense sociale, pour lequel il s'agit avant tout de mesurer un délit (sans distinction) à l'aune du danger qu'il fait courir à une société implicitement représentée comme consensuelle et ontologiquement digne d'être défendue - inflexion nette vers un absolutisme de la conception de l'exercice du gouvernement.

12 Au fil du temps et jusqu'à nos jours, la justification de l'existence même d'un traitement de faveur pour le délit politique n'a cependant pas été fondamentalement remise en cause ; cependant, si le principe demeure intact, des conditions et exceptions de plus en plus nombreuses ont progressivement restreint l'extension de cette catégorie aux infractions les plus manifestement et directement politiques, au point que l'application de ce principe est devenue rarissime. De façon indicative, C. Panier intitule en 1977 l'article qu'il consacre au sujet «la déliquescence de la notion de délit politique ${ }^{15}$ ». Toutefois, pour réduite qu'elle soit, la qualification politique d'un délit demeure théoriquement possible en droit belge : la jurisprudence très restrictive de la Cour de cassation, même si elle ne laisse qu'une marge étroite, réaffirme par là même l'existence de la notion de délit politique et son applicabilité ${ }^{16}$.

Cependant, la loi du 19 décembre 2003 relative aux infractions terroristes marque, en la matière, un véritable tournant. En effet, à la suite de la décision-cadre du Conseil de l'Union européenne du 13 juin 2002, le Parlement belge a adopté une loi insérant dans le Code pénal une série de nouvelles infractions appelées "terroristes » dont la définition, et plus précisément l'élément intentionnel spécifique, semble entrer en contradiction avec le statut privilégié que la Constitution octroie au délit politique et remet radicalement en question son existence même.

L'incrimination du terrorisme en Belgique : une histoire récente

14 Depuis la fin des années 1990, l'Union européenne a construit l'objet «terrorisme » comme l'un des thèmes centraux de son action en matière de justice et d'affaires 
intérieures ${ }^{17}$, à côté de l'immigration, de la criminalité organisée et du trafic de stupéfiants. Les attentats du 11 septembre 2001 ont poussé le Conseil, sous présidence belge, à anticiper les négociations et l'adoption d'une proposition de décision-cadre préparée par la Commission et fin prête depuis le 5 septembre. En effet, l'adoption de cette décision-cadre avait été initialement fixée à l'agenda de la présidence espagnole, qui devait succéder à la Belgique à la tête de l'Union et qui tenait, pour des raisons de politique intérieure, à ce que son mandat soit associé à la concrétisation de la décisioncadre relative à la lutte contre le terrorisme ainsi que de celle relative au mandat d'arrêt européen. Soucieux d'adresser « un signal fort » aux citoyens de l'Union, selon les termes du représentant belge au sein du CATS $^{18}$, le Conseil charge le CATS et le COREPER de retravailler le texte de la Commission et d'avancer au maximum sur la voie d'un accord. Activement dirigées par une présidence belge multipliant les propositions écrites de compromis ${ }^{19}$, les différentes délégations s'accordent rapidement, si bien que le texte est pratiquement terminé dès la fin du mois de novembre 2001, et il est finalement adopté le 13 juin 2002. Le statut juridique particulier de cet instrument de droit européen ${ }^{20}$ est destiné à imposer aux Etats un certain rapprochement de leurs législations pénales respectives, censé permettre en l'espèce de lutter plus efficacement contre la «menace terroriste» en facilitant la coopération judiciaire et policière. Cependant, conformément au texte du TUE, ce rapprochement est limité aux objectifs fixés par la décision-cadre, mais ne lie pas les Etats quant à la forme ou aux moyens, laissés à la discrétion des Etats membres afin de garantir la cohérence interne des systèmes juridiques nationaux et de ne pas menacer l'autonomie des Etats en ces matières sensibles.

Un nouvel « objet » : le terrorisme

Dans la loi du 19 décembre 2003 « relative aux infractions terroristes », le législateur belge a pourtant choisi d'épouser au plus près les formules retenues par la décision-cadre en effectuant une retranscription scrupuleuse d'une bonne partie de celle-ci dans son Code pénal, et notamment les nouvelles infractions terroristes et leur définition, contrairement au choix de l'Allemagne, de l'Espagne, de la France, de la GrandeBretagne, de l'Italie et du Portugal, notamment. En effet, à la différence de ces derniers, la Belgique ne disposait pas d'une législation pénale antérieure spécifique au terrorisme; nonobstant les attentats et faits de violence qualifiés de «politiques » qui ont agité le pays dans les années 1980 - notamment les plasticages d'installations militaires et civiles par les CCC et la mise sur pied d'une Commission d'enquête parlementaire sur le terrorisme et le grand banditisme suite à l'affaire des « tueurs du Brabant "- le législateur belge n'a pas jugé utile de se doter d'un arsenal législatif particulier pour réprimer le terrorisme, dont le concept même n'est alors nulle part défini en droit belge sensu lato. Il faut attendre 1992 pour relever la première définition proprement juridique du terrorisme, qui apparaît dans un champ particulier a priori étranger à la question du maintien de l'ordre public : le droit assuranciel. En effet, c'est un arrêté royal d'exécution de la loi sur les assurances terrestres du 25 juillet 1992 qui définit, parmi les risques couverts par les polices d'assurance, l'acte de terrorisme comme étant : «[1'] action organisée dans la clandestinité à des fins idéologiques, politiques ou sociales, exécutée individuellement ou en groupe et attentant à des personnes ou détruisant un bien [...] en vue d'impressionner le public et de créer un climat d'insécuritée ${ }^{21}$ ».

16 Six ans plus tard, le Parlement porte sur les fonds baptismaux la nouvelle loi organique des services de renseignement et de sécurité, qui établit les grandes lignes de l'organisation et des missions de la Sûreté de l'Etat, parmi lesquelles figure le 
terrorisme. L'article 8 de cette loi stipule qu'il faut entendre par «terrorisme le recours $\grave{a}$ la violence à l'encontre de personnes ou d'intérêts matériels, pour des motifs idéologiques ou politiques, dans le but d'atteindre ses objectifs par la terreur, l'intimidation ou les menaces ${ }^{22}$ ”. Si la portée de cette définition mérite certainement d'être discutée, cette dernière ne conditionne pourtant que l'activité de police politique exercée par les services de renseignement, dont les fonctions sont traditionnellement distinctes de la police judiciaire ou administrative, et dont la justification dans le cadre d'une société démocratique doit être débattue dans son propre cadre de référence qui dépasse nos préoccupations directes dans le cadre de cet article ${ }^{23}$. En revanche, il est utile de relever que cette définition est aujourd'hui toujours pertinente, pour la Sûreté de l'Etat, alors même que le système pénal possède depuis 2003 son propre point de référence en la matière à travers les nouveaux articles 137 à 141 ter du Code pénal.

La loi du 19 décembre 2003 relative aux infractions terroristes

17 Adopté par le Parlement après des débats particulièrement restreints, le texte établit trois nouvelles infractions: la participation aux activités d'une organisation terroriste et sa direction font l'objet d'une sanction pénale particulière, mais s'appuient sur la définition des infractions terroristes proprement dites donnée à l'article 137 du Code pénal, que nous examinerons plus en détail ci-après. Outre ces infractions, la loi énonce des dispositions relatives aux peines assorties à ces nouveaux délits, qui font l'objet d'une majoration systématique par rapport aux délits de droits communs en raison de l'intention particulière de l'infraction terroriste. Ensuite, d'autres dispositions traitent de la participation, de la direction et du financement d'activités terroristes, excluent les conflits armés du champ d'application de la loi, rappellent la primauté des droits fondamentaux et, enfin, étendent aux nouvelles incriminations la possibilité du recours à des techniques particulières d'enquête.

18 L'article 137 du Code pénal constitue le cœur de ce nouveau dispositif. Etablissant les critères juridiques de qualification des infractions terroristes, autrement dit leur définition, il est également l'élément clé de la compréhension de ce dispositif en ce qu'il donne à voir la construction de son concept central par le pouvoir législatif. Concrètement, l'article 137 établit les deux éléments constitutifs des infractions terroristes :

19 - un élément matériel. Fixé par les paragraphes 2 et 3 de l'article 137, cet élément matériel est constitué de deux listes fermées d'infractions figurant déjà dans le Code pénal ( $(2)$ ou insérées pour l'occasion ( $(3)$. Si le paragraphe 2 renvoie de ce fait explicitement à des dispositions précises du Livre II du Code pénal ${ }^{24}$ et donc à des comportements déjà passibles de poursuites judiciaires ${ }^{25}$, le paragraphe 3 suscite une première réflexion, car il énumère une série d'infractions jugées, selon les termes même de l'exposé des motifs de la loi, «non-poursuivies ou insuffisamment poursuivies » par le droit pénal en vigueur ; par conséquent, cela implique que des actes jusqu'ici non infractionnels ou assortis d'une sanction pénale modérée sont inclus dans le champ d'application matériel de la qualification «terroriste ». Ainsi, d'aucuns ${ }^{26}$ craignent que certaines actions, certes illicites, mais ordinairement considérées d'un œil sinon indulgent, à tout le moins plus favorable, et ce en raison notamment de leur inscription traditionnelle dans l'expression d'une protestation ou de revendications syndicales, sociales ou politiques, puissent dorénavant tomber sous l'acception de terrorisme ${ }^{27}$.

20 - un élément moral. Défini au paragraphe $1^{\text {er }}$ de l'article $137^{28}$, il se compose de deux éléments distincts, relatifs d'une part aux effets de l'acte et d'autre part à l'intention de 
son auteur, tous deux qualifiés par des formulations supposées limiter la portée de l'incrimination. Le premier est relatif à l'évaluation des conséquences potentielles de l'infraction, tandis que le deuxième porte sur l'intention de l'auteur et établit, à ce titre, le véritable critère de discrimination des infractions terroristes par rapport aux infractions de droit commun qui en constituent l'élément matériel.

L'intention politique couverte par l'intention terroriste

Il est utile à ce stade de revenir sur quelques-unes de nos considérations antérieures et d'opérer une comparaison éclairante. Comme défini par la Cour de cassation, qui en a progressivement restreint la portée, un délit n'est politique que «si, eu égard à la nature même de l'infraction, il consiste en une atteinte portée directement à l'existence, à l'organisation ou au fonctionnement des institutions politiques; ou s'il a été commis dans le but de porter une telle atteinte aux institutions politiques et que, vu les circonstances particulières de sa commission, le fait entraîne ou peut entraîner directement une telle atteinte [... $]^{29}$ ». Or, si l'on ramène cette définition à celle de l'élément intentionnel des infractions terroristes, force est de constater que les deuxième (contrainte exercée sur un pouvoir public) et troisième (déstabilisation ou destruction des structures fondamentales d'un pays, notamment politiques) critères de qualification sont très proches de ceux fixés par la jurisprudence en matière de délit politique, et les inclut sans aucun doute. En revanche, l'infraction terroriste peut être établie à partir des effets potentiels de l'acte incriminé évalués en fonction de leur nature et de leur contexte, tandis que la qualification d'une infraction comme délit politique nécessite une atteinte directe aux institutions politiques.

Depuis le 19 décembre 2003, le droit pénal belge offre donc, en matière de délits politiques, une perspective quelque peu paradoxale. En effet, puisqu'il semble bien, en comparant leurs définitions respectives, qu'un délit politique figurant dans la liste des infractions reprise à l'article 137, $\S 2$ et 3 constitue a fortiori une infraction terroriste, on doit en conclure qu'en matière d'intention politique, le législateur belge présente désormais deux appréciations antagonistes : au régime de faveur inscrit dans les textes fondateurs de l'Etat peu après la Révolution belge de 1830 - régime dont la restriction des conditions d'exercice n'a jamais atteint le principe lui-même - se superpose la qualification "terroriste " dont l'élément constitutif le plus propre, qui définit une intention politique sensu lato, justifie au contraire une répression plus sévère.

L'intention politique : pivot d'un retournement

23 L'évolution de la conception de l'action politique illégale par le législateur belge aboutit dès lors à une situation apparemment confuse de coexistence entre deux perceptions opposées de l'intention politique. Cependant, dans une perspective diachronique, cette évolution peut être lue comme évocatrice des changements intervenus dans le mode d'exercice du gouvernement et, dès lors, comme indicative des bouleversements pressentis dans la gouvernementalité contemporaine.

L'intention politique, du délit politique au terrorisme : une histoire en trois temps Premier temps : le gouvernement libéral. Institué dès l'origine par la Constitution, le régime spécifique dont jouit le délit politique en Belgique et le souci même de confier au jury populaire, et de façon plus générale à la jurisprudence, le soin d'en tracer les limites est la concrétisation des conceptions libérales et de l'esprit révolutionnaire de l'époque qui marquent une «tendance à considérer avec moins de rigueur les infractions politiques ${ }^{30}$ ».

Deuxième temps: le gouvernement conservateur. Rapidement cependant, la consolidation de l'Etat belge et la stabilité politique acquise entraînent le développement d'une 
idéologie plus conservatrice, soucieuse du maintien de l'ordre établi, mais variant selon les périodes en fonction de l'évolution de la pensée juridique, dont les arrêts successifs de la Cour de cassation définissant le délit politique se font l'écho ${ }^{31}$. Cependant, le mouvement de restriction de la portée de cette définition, jamais démenti, aboutit au fil du temps à une quasi inapplicabilité de fait de la qualification politique d'un délit en raison du caractère exclusif des critères établis par la jurisprudence. Toutefois, le principe même de la spécificité du délit politique en raison de la noblesse de ses mobiles demeure, indépendamment des conditions de son application, comme le laisse apparaître cet exemple tiré d'un précis de droit pénal paru en Belgique en 1946 :

«la détention est destinée à réprimer les attentats politiques. Ces attentats supposent, en général, moins de perversité que d'audace, moins de corruption que d'inquiétude. L'amélioration morale a beaucoup moins à faire qu'en matière de droit commun ${ }^{32}$ ».

Troisième temps: le gouvernement "libéral avancé»? Changement radical en décembre 2003, où le Parlement établit par le truchement de l'incrimination des actes terroristes une nouvelle référence en matière d'intention politique: prenant implicitement le contre-pied d'un principe vieux de plus de cent soixante-dix ans, il consacre dans des termes très larges et pour une grande variété d'infractions la dévalorisation de l'intention politique, en l'assortissant de peines aggravées par rapport aux infractions de droit commun. Dans les termes de l'article $137, \S 1^{\mathrm{er}} \mathrm{du}$ Code pénal, commettre l'une des infractions visées aux $\S \S 2$ et 3 «dans le but d'intimider gravement une population ou de contraindre indûment des pouvoirs publics ou une organisation internationale à accomplir ou à s'abstenir d'accomplir un acte, ou de gravement déstabiliser ou détruire les structures fondamentales politiques, constitutionnelles, économiques ou sociales d'un pays ou d'une organisation internationale» est dorénavant tenu pour plus condamnable que toute autre motivation.

Schématiquement, on peut représenter cette évolution comme suit :

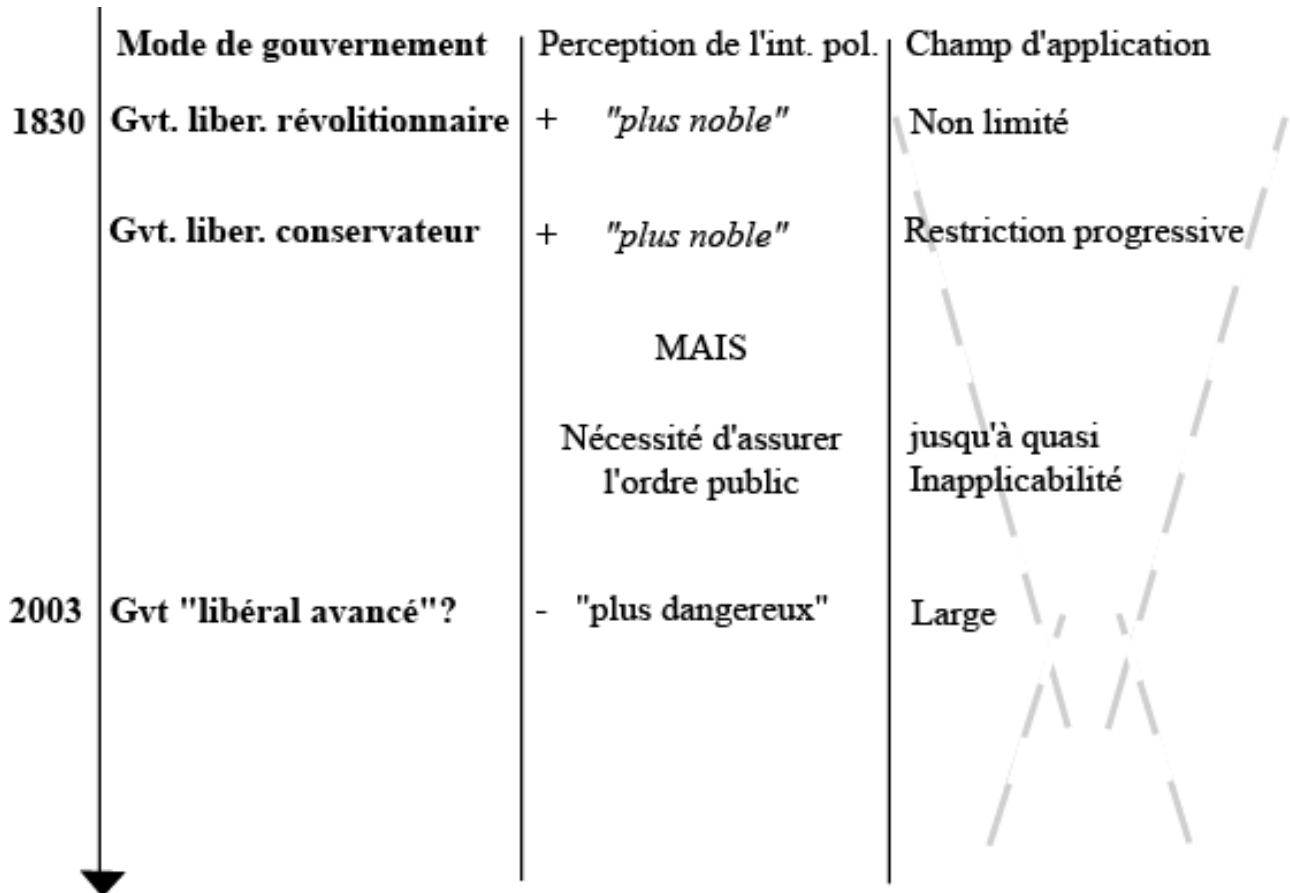

Les différents modes de gouvernement (première colonne) sont caractérisés par une perception particulière de l'intention politique de l'infraction par rapport aux délits de 
droit commun (deuxième colonne) et par une extension variable du champ d'application matérielle des infractions caractérisées et définies par cette intention politique (délit politique et terrorisme). Les traits discontinus en grisé dans la troisième colonne offrent une représentation visuelle de l'évolution de ce champ d'application en fonction de la perception de l'intention politique : lorsque cette dernière est considérée comme «noble » (délit politique), ce champ d'application est peu à peu restreint; lorsqu'elle est tenue pour «vile» (terrorisme), ce champ d'application est fortement élargi.

Cette représentation synthétique suscite trois observations majeures: primo, la scansion de la temporalité en trois modèles de gouvernement ne prétend aucunement établir un quelconque canevas chronologique objectif. Les trois modèles proposés ne sont que l'étiquetage de modes de construction juridique et de conception du rôle du législateur, et, plus largement, du rôle du politique en matière pénale, dont les contours des deux premiers n'ont été que très grossièrement esquissés dans le cadre de cet article, et dont les caractéristiques du troisième seront brièvement évoquées à titre d'hypothèse en conclusion. De même, ce schéma n'apporte aucun éclairage sur les interactions entre ces modèles (succession exclusive, coexistence, superposition, etc.) ni sur le moment historique de leur émergence ou de leur disparition, contrairement à ce que pourrait induire le mode de présentation retenu.

Secundo, la transition entre l'appréciation favorable de l'intention politique et sa dépréciation est assurée par la restriction du champ d'application effective du régime de faveur : le principe établi en 1830 est progressivement vidé de sa substance, de sorte que sa contradiction établie au cœur même de l'incrimination du terrorisme n'a guère soulevé de résistance. Davantage, on relèvera que le passage de l'appréciation à la dépréciation de l'intention politique s'accompagne d'une expansion brutale du champ d'application matérielle de la qualification: une infraction commise dans un but politique sensu lato ne constituera que très exceptionnellement un "délit politique " alors qu'elle sera beaucoup plus certainement qualifiée de " terroriste ».

31 Tertio, et plus essentiellement, il semble que la restriction progressive, puis le retournement dans l'appréciation de l'intention politique soient liés à l'émergence et au développement d'une conception du gouvernement démocratique comme garant de l'ordre public. En fait, la « démocratie » demeure la référence ultime dans l'exercice du gouvernement depuis la naissance de la Belgique, mais cette référence consensuelle dissimule des acceptions profondément divergentes en fonction des époques, bien que non exclusives: le droit des peuples à disposer d'eux-mêmes et les libertés fondamentales sacralisées par l'Assemblée constituante sont peu à peu tempérés par l'émergence d'une préoccupation pour le maintien du mode de gouvernement exercé et auto-défini comme démocratique. Autrement dit, l'assimilation des conditions démocratiques de gouvernement avec les conditions de gouvernement en vigueur en Belgique tend progressivement à l'exclusivité : si le gouvernement belge en place est un gouvernement démocratique, le seul gouvernement démocratique est le gouvernement belge en place. Dans les faits, les différentes phases de cette mutation de la représentation du gouvernement par lui-même sont visibles à travers le développement croissant des exceptions au régime de faveur, dont bénéficient théoriquement les délits politiques, "en vue de l'adapter aux nécessités effectives de la protection de l'Etat ${ }^{33}$ ", jusqu'au retournement introduit en 2003 par la législation antiterroriste.

Une représentation de la démocratie absolue? 
32 L'évolution de la qualification de l'intention politique en Belgique renvoie l'Etat à sa propre conception de lui-même en tant que forme ultime de gouvernement démocratique. A travers la dépréciation progressive de l'intention politique du délit, le pouvoir politique belge contemporain est l'héritier d'une auto-identification progressive de l'exercice contingent et aléatoire du gouvernement aux principes démocratiques, d'un arraisonnement de la chose publique par l'ordre public, d'une réduction du champ de la pratique politique au cadre étatique et aux formes de la participation démocratique légitime. La démocratie est identifiée au respect de ses conditions formelles, le changement démocratique ne peut intervenir qu'à l'intérieur du cadre existant, et donc dans des formes maîtrisées par le gouvernement en place. La relation historique entre délit politique et terrorisme nous renvoie au champ des conditions de vérité du discours, des conditions de ce qui est susceptible d'être débattu, de ce que Michel Foucault appelle « la politique du vrai ${ }^{34}$ ».

Dépolitisation du terrorisme... ou du politique?

L'incrimination du terrorisme en Belgique ne peut, selon nous, être assimilée à une dépolitisation que dans un sens particulier : celui d'une expulsion par la politique d'une partie du champ $d u$ politique. En s'auto-identifiant comme forme aboutie du gouvernement démocratique, le gouvernement étatique contemporain exclut ipso facto du champ politique dont il établit les limites, toute mise en question de son existence ou des formes de son exercice, réduisant ainsi le champ politique au champ politique légitime. En d'autres termes, il conditionne l'accès au champ politique au respect des formes par lui établies et qui lui assurent sa perpétuation, et soumet de la sorte l'alternative politique au règne du même. Cependant, cela suppose de tirer du caractère le plus radicalement politique des formes proscrites la justification même de leur dépolitisation, sortant pour ainsi dire du champ politique par excès. Concept " creux » se prêtant à merveille à l'identification du changement au danger et alimentant les spéculations les plus incertaines, le terrorisme, en tant qu'objet consensuel de rejet, permet d'accomplir cette dépolitisation au prix d'une disqualification du politique. Ainsi, en Belgique, mais aussi pour l'Union européenne, l'intention de déstabiliser gravement les structures fondamentales (et notamment économiques) d'un pays ou d'une organisation internationale représente le danger ultime pour les sociétés démocratiques, ce qui semble indiquer à l'évidence que lesdites structures sont consubstantielles au régime démocratique, et qu'il n'est donc pas de démocratie possible hors de ces structures. De même, il est désormais plus condamnable de commettre une infraction dans un but politique que dans un but de lucre, ce dernier constituant au fond le principe ultime guidant l'existence des individus dans les sociétés libérales avancées.

Le terrorisme et la conception de la société démocratique

Dans un précédent numéro de Cultures \& Conflits ${ }^{35}$, D. Bigo, dans l'article qu'il consacrait à la mondialisation du discours sur l'insécurité, ne manquait pas d'attirer l'attention du lecteur sur la relation entre les places respectives qu'y occupent le terrorisme et la violence politique :

« ainsi, dans ces discours d'experts [en matière de sécurité], la notion de violence politique, au cœur du sujet, n'apparaît nulle part ou presque dans les commentaires. Elle est remplacée par celle de terrorisme global occultant la dimension du politique. [...] Les confusions de ces analyses [d'experts] dites stratégiques portent, pour ne citer que les plus flagrantes, sur le rapport entre sécurité de l'Etat et sécurité personnelle, sur la relation entre menace de défense, 
menace policière, risque pour la société et changement social. Il en résulte la plupart du temps une incompréhension du social, avec une "stratégisation" des dangers du quotidien, une insécurisation du monde où le risque est toujours lu comme danger et non comme opportunité ${ }^{36} »$.

Rapporté à notre objet empirique, cet extrait garde toute sa pertinence si l'on substitue aux « experts » le législateur belge et, plus largement, le champ du discours politique sur le terrorisme en Belgique : cela suggère donc que certains traits de l'évolution du discours en matière de délit politique, que nous avons brièvement identifiés dans ces lignes, peuvent être également observés dans d'autres champs empiriques et discursifs, renvoyant à des approches criminologiques plus globales, et relatives au rôle du système pénal dans le maintien de l'ordre social dans nos sociétés contemporaines ${ }^{37}$.

Cela pourrait également attirer l'attention sur le relatif désintérêt de la criminologie francophone pour la création de la loi et les questions les plus directement liées à la place du pénal dans des enjeux politiques plus globaux, et susciter un intérêt pour un rapprochement interdisciplinaire avec les sciences politiques, qui offrent au criminologue un autre regard sur son objet. Ainsi les propos d'E. Ferri, membre illustre de l'école positiviste italienne compromis dans le régime fasciste, nous incitent-ils à la réflexion sur la transformation du mode de gouverner et sur ses axiomes implicites, en ce qu'ils nous permettent de prendre la mesure des changements de paradigme intervenus en moins d'un siècle en matière de construction pénale du terrorisme : «[...] la criminalité évolutive, inspirée de mobiles altruistes et sociaux, vise l'ordre politico-social établi, ordre qui est toujours historiquement transitoire. La répression de la criminalité atavique seule constitue une protection de la collectivité, la répression de la criminalité évolutive ayant simplement comme but la défense de la classe sociale dominante ${ }^{38}$ ".

Le caractère suranné de la nosographie et des catégories utilisées a un mérite incontestable : celui de nous rappeler que la vérité du discours, qu'il soit scientifique ou politique, est elle aussi transitoire.

La démocratie par l'Etat « libéral avancé » et la gouvernementalité

38 A partir d'une analyse empirique locale portant sur l'adoption de nouvelles incriminations du terrorisme en Belgique, nous nous sommes concentrés sur l'élément intentionnel « terroriste » tel qu'il figure dans la loi belge du 19 décembre 2003 et dans la décision-cadre de l'Union européenne du 13 juin 2002 qui l'a inspirée et nous l'avons replacé dans le contexte plus large de l'évolution du statut légal du délit politique. Nous en avons dégagé trois modèles schématiques de gouvernement - libéral révolutionnaire, libéral conservateur, "libéral avancé " - correspondant à trois conceptions de l'opposition politique et, plus largement, de la démocratie. Bien que les deux premiers modèles appellent sans aucun doute de plus amples développements, le processus contemporain d'émergence d'un hypothétique modèle " libéral avancé », aux contours encore très incertains, doit être cerné avec davantage de précision : à partir de l'analyse empirique qui précède, nous pouvons avancer trois principes sous-jacents reliés entre eux et qui semblent marquer une certaine rupture dans l'exercice de la gouvernementalité en Belgique, mais également au niveau de l'Union européenne ${ }^{39}$.

39 A travers le dispositif que constitue la loi du 19 décembre 2003 et l'évolution de la (dis-)qualification $\mathrm{du}$ délit politique, il semble que cette gouvernementalité contemporaine se saisisse d'elle-même comme une triple fin :

40 - fin de l'évolution démocratique. Comme nous l'avons relevé plus haut, l'Etat s'octroie implicitement le statut de démocratie achevée; cela signifie donc que le changement 
politique est établi comme paradoxal, en ce qu'il ne peut remettre en cause le mode de gouvernement que dans les limites par lui imposées et qui garantissent la continuité de ce mode. Autrement dit, le changement politique démocratique est celui qui garantit l'immutabilité des formes du gouvernement, tandis que celui qui, à l'inverse, vise les «structures fondamentales» de la gouvernementalité ne peut être qu'antidémocratique.

41 - fin des idéologies. Cette auto-identification du mode de gouvernement contemporain avec les principes démocratiques se lit également comme une prétention à constituer un seuil minimal de valeurs communes, en deçà des divergences d'orientation politique : en quelque sorte, les orientations idéologiques, entendues comme portant sur l'enjeu «de désigner à grands traits le sens véritable des actions collectives, de dresser le modèle de la société légitime et de son organisation, d'indiquer simultanément les détenteurs légitimes de l'autorité, les fins que doit se proposer la communauté et les moyens d'y parvenir ${ }^{40} 》$, sont désormais exclues du champ politique légitime.

42 - fin de l'action politique illégale. D'un point de vue plus particulier, le contexte général ainsi défini entraîne la fin du statut privilégié accordé au délit politique, qui n'est plus nécessaire en raison de l'évolution dans la représentation de la gouvernementalité contemporaine et ce à deux niveaux : au niveau de la forme, en ce que la démocratie «libérale avancée » considère que tout changement politique légitime est accessible par des moyens légaux - démocratie achevée ; au niveau du fond, en ce que les actions illégales d'opposition idéologique sont de facto antidémocratiques - valeurs communes.

43 Analysé sous un angle constructiviste radical inspiré des travaux portant sur la néogouvernementalité, chacun de ces traits fait apparaître une perspective plus générale :

44 - démocratie formelle : la gouvernementalité « libérale avancée » opère une réduction de la démocratie aux formes démocratiques actuelles. En cela, elle permet l'auto-affirmation perpétuelle (ou, tout du moins, une hétéro-affirmation interne aux Etats mutuellement reconnus-affirmés démocratiques) en tant que seule forme démocratique légitime; cette absolutisation, cet effacement de la contingence des formes actuelles du gouvernement a pour corollaire la dis-qualification de l'intention politique « idéologique » à travers la figure du terrorisme, qui justifie les exceptions aux droits démocratiques dans la mesure où ils menacent la démocratie elle-même. L'Etat « libéral avancé » est le dépositaire, le garant et la concrétisation indépassable de la démocratie. A cet égard, l'objet «terrorisme » est la manifestation la plus visible d'une évolution plus générale de la gouvernementalité observée par D. Garland en ces termes : « il y a un relâchement dans l'attention accordée aux libertés civiles des suspects, et les droits des prisonniers, [...]. A la demande de protection contre l'Etat s'est substituée de manière croissante la demande de protection par l'Etat ${ }^{41} »$.

45 - consensus mythique implicite : la démocratie - i.e. le mode de gouvernement exercé par les Etats identifiés comme démocratiques - explicitement liée aux droits fondamentaux et notamment à la liberté de pensée est associée implicitement et nécessairement à l'économie capitaliste, au modèle étatique (c'est-à-dire au respect des limites et du mode de gouvernement des Etats du «club» démocratique) et à la «culture occidentale » - sans plus aucune pertinence géographique, le terme reste employé, son caractère indéfini permettant une inclusion à géométrie variable en fonction des circonstances - qui constituent les conditions du discours politique légitime. Victor et Aaron Kappeler remarquent, à propos de la construction du terrorisme comme ennemi de la civilisation et de la liberté, que "[cette] construction dénie la possibilité d'une 
perspective différente, établissant que la base du conflit est la liberté plutôt qu'une idéologie politico-économique concurrente $e^{42}$ ». Toute critique de l'un de ces trois axiomes "méconnus $^{43}$ » est, en conséquence, contraire à la démocratie, a fortiori si cette critique s'accompagne d'actes illégaux.

- Réduction du champ de l'opposition politique aux formes maîtrisées et imposées. La conséquence de ces observations est la limitation de l'opposition politique légitime au non-changement des formes du gouvernement méconnues comme contingentes (gouvernement étatique, capitalisme, occidentalisme); cette «a-politisation» de l'action politique s'accompagne d'une "politisation» des questions pénales, dans le sens que David Garland accorde à ce terme à propos de l'évolution des mesures pénales aux Etats-Unis et en Grande-Bretagne : «la "politisation" suggère parfois une polarisation des positions, mais la forme populiste que la politique pénale a prise a eu exactement l'effet inverse. Loin d'une différenciation des positions politiques, ce qui a finalement émergé, dans les années 1980 et 1990, est un rétrécissement du débat et une convergence remarquable des propositions de tous les principaux partis politiques ${ }^{44}$ ». Appliquée à l'objet « terrorisme », la gestion politique sensu lato ne sort pas du positivisme étiologique, et appelle pour les plus autoritaires un contrôle préventif global des populations-cibles, et, pour les plus progressistes, une extension du modèle démocratique et de ses implicites au reste du globe.

Cette distinction entre " politique » et "idéologie », ou plutôt l'exclusion de la seconde hors du champ de la première, se donne à voir avec une acuité particulière dans la construction de l'objet « terrorisme ». Le point de départ indépassable, le terminus a quo du questionnement est figé sur la représentation d'une menace fondamentale pour la démocratie, existant en soi et portant sur les fondements mêmes de la gouvernementalité. Le terrorisme en tant que figure de l'ennemi renvoie à cette sortie de l'idéologie et à ce consensualisme en matière de contrôle. Si l'on situe, avec Garland, les premières manifestations de l'émergence de cette nouvelle gouvernementalité à la fin des années 1970, on peut relever que c'est également à cette époque que la figure du « communisme ennemi de la démocratie » a commencé à perdre de sa prévalence. Or, le cœur de cette opposition entre communisme et sociétés libérales occidentales était profondément et radicalement idéologique, le terrorisme ne constituant qu'un moyen. Aujourd'hui, l'indiscrimination de la définition du terrorisme, supposée rendre compte de son "caractère protéiforme ", marque surtout l'expulsion de l'idéologie hors du champ politique. En effet, quand il est qualifié autrement que de manière tautologique, le terrorisme est souvent rapproché, de manière plus ou moins explicite, de l'islam radical, et, plus rarement, de mouvements contestataires en matière politique, économique ou sociale. Dès lors, il est soit réduit à des questions de culture ou de religion, soit identifié à de la délinquance ou à de l'insubordination de la part de jeunes fauteurs de troubles. En conséquence, ce qui aura été identifié comme «terrorisme » se verra inexorablement vidé de sa dimension idéologique.

Résistances? Contre-conduites

Pour Michel Foucault, la gouvernementalité, en tant qu'art de conduire les conduites, au sens général, et en tant que forme contemporaine de cet art, au sens particulier, amène à poser autrement la question de la résistance. En effet, cette gouvernementalité ne repose que minoritairement sur la contrainte (objectivation), et s'appuie surtout sur l'obéissance "spontanée " (subjectivation) des individus pour gérer les populations. Parmi les dispositifs mobilisés à cet effet, la loi contribue à transformer une 
contingence politique en nécessité naturelle, et à faire en l'espèce de l'objet « terrorisme » qu'elle construit une réalité indépendante du processus de qualification pénale dont elle est l'aboutissement.

Dès lors, il nous semble que la question des résistances doit être posée en tant que recherche de "contre-conduites ${ }^{45}$ " répondant à l'évolution de la gouvernementalité. Ainsi, et en nous limitant aux quelques éléments esquissés dans ce texte, une contreconduite pertinente doit passer par une «repolitisation", c'est-à-dire une reconnaissance des implicites du modèle démocratique actuel et du consensus anidéologique apparent que l'objet «terrorisme » contribue à établir. Pour les acteurs du monde scientifique en particulier, il nous semble que le développement d'une approche radicalement constructiviste du terrorisme (et, plus généralement, des nouvelles occurrences de la réalité), qui sorte du modèle étiologique et qui se centre sur les processus de production de l'objet, peut contribuer à développer un schème d'intelligibilité du modèle de gouvernementalité contemporaine et, qui sait, alimenter ainsi le développement d'un activisme adapté, pertinent et efficace: un activisme « néo-gouvernemental»?

\section{NOTES}

1.. Les Cellules communistes combattantes commettent leur premier attentat à la bombe le 2 octobre 1984 ; elles en commettront près de trente sur le même modèle, à savoir la destruction de cibles stratégiques ou symboliques (installations militaires, entreprises américaines, banques, etc.) en ayant au préalable prévenu les autorités et les personnes sur place afin d'éviter tout dommage physique. Cependant, en avril 1985, deux pompiers bruxellois sont victimes d'une erreur de coordination et décèdent dans l'explosion précoce d'une voiture piégée. Les quatre membres identifiés de ce groupe ont été arrêtés en 1986 et condamnés, le dernier d'entre eux encore emprisonné a été libéré en 2003.

2.. "Toute association formée dans le but d'attenter aux personnes ou aux propriétés est un crime ou un délit, qui existe par le seul fait de l'organisation de la bande », Art. 322 du Code pénal belge.

3.. Au sens foucaldien du terme.

4.. Voir Tulkens F., van de Kerchove M., Introduction au droit pénal, Bruxelles, StoryScientia, 1997, p. 269.

5.. Ibid.

6.. Ibid.

7.. Ibid., p. 270.

8.. Les autorités belges ne connaissent que trop bien les effets de contagion des mouvements révolutionnaires français, qui ont porté la Révolution belge.

9.. Selon van den Wijngaert C., «[...] le délit politique se compose d'un élément subjectif [dans l'intention de son auteur] et d'un élément objectif [par son effet] » ( het politieke misdrijf bestaat uit een subjectief en een objectief element ») van den Wijngaert C., Strafrecht en Strafprocesrecht, Anvers, Maklu, 1998, p. 153. 
10.. Tulkens F., van de Kerchove M., op. cit., p. 274.

11.. Par exemple, « les usurpations illicites de la qualité d'électeur tendent à troubler l'exercice régulier du système électoral et à compromettre les droits constitutionnels par lesquels les citoyens participent au gouvernement du pays; et qu'elles portent ainsi directement atteinte à une de ses institutions fondamentales ", Cass., 19 août 1970.

12.. Ces circonstances et conditions ont été fixées de manière très restrictive par la Cour de cassation, à tel point que le dernier délit politique mixte reconnu en Belgique remonte à février 1900.

13.. «A partir du XXe siècle, une certaine réaction s'amorce dans la manière d'envisager le délit politique qui apparaît à nouveau comme justifiant une répression plus sévère », Tulkens F., van de Kerchove M., op. cit., p. 269.

14. Pour E. Ferri et $\mathrm{C}$. Lombroso les délits politiques font partie de la « criminalité évolutive » qui « tend à hâter les phases futures de la vie politico-sociale », Papadatos P., Le Délit politique, Genève, Librairie E. Droz, 1955, p. 46.

15.. Panier C., « La déliquescence de la notion de délit politique », Contradictions, 1977, pp. 3 et suivantes.

16.. «Un délit ne peut constituer un délit politique que s'il résulte nécessairement de la nature même du délit qu'il consiste en une atteinte portée directement à l'existence, à l'organisation ou au fonctionnement des institutions politiques ou qu'il a été commis dans le but de porter une telle atteinte aux institutions politiques et que le fait, vu les circonstances particulières de sa commission, a ou peut avoir directement une telle atteinte pour conséquence. »Cour de cassation, 18 novembre 2003, RG P.03.0487.N.

17.. L'acronyme JAI, qui s'est imposé depuis le Traité de Maastricht en 2002, est progressivement abandonné en raison de l'évolution constitutionnelle de l'Union, qui lui a substitué l'Espace de liberté, de sécurité et de justice (ELSJ) par le Traité d'Amsterdam de 1997.

18.. Le Comité de l'Article trente-six (CATS) est un organe chargé de préparer les travaux du Conseil dans les matières JAI ; il est subordonné au Comité des représentants permanents (COREPER), qui exerce également une fonction de préparation des décisions du Conseil. Dans les faits, la plupart des décisions du Conseil en la matière se prennent au sein du CATS et du COREPER.

19.. Les entretiens réalisés avec plusieurs hauts fonctionnaires belges en charge du dossier au niveau national ou au sein de la délégation auprès de l'Union ont mis en évidence le rôle de la Belgique dans ces négociations : le rôle médiateur de la présidence s'est en effet concrétisé par la rédaction de nombreuses propositions écrites visant à concilier les positions divergentes, mais intégrant également les objectifs et intérêts propres à la délégation belge ; le résultat le plus visible de cette influence de la Belgique sur le contenu de la décision-cadre à travers le jeu des négociations est la conservation du caractère facultatif du recours aux « repentis » en matière de terrorisme, qu'une majorité d'Etats voulait rendre obligatoire (art. 6 de la décisioncadre du Conseil de l'Union européenne du 13 juin 2002 relative à la lutte contre le terrorisme).

20.. Voir art. 31,e) TUE (Traité de l'Union européenne). La décision-cadre est un instrument ad hoc pour le troisième pilier, et vise explicitement à imposer un rapprochement des législations dans les matières qui y sont soumises.

21.. Arrêté royal réglementant l'assurance contre l'incendie et d'autres périls, $L e$ Moniteur belge, 31 décembre 1992. 
22.. De façon plus complexe et complète, les services de renseignement et de sécurité ont notamment pour mission « de rechercher, d'analyser et de traiter le renseignement relatif à toute activité qui menace ou pourrait menacer la sûreté intérieure de l'Etat et la pérennité de l'ordre démocratique et constitutionnel, la sûreté extérieure de l'Etat et les relations internationales, le potentiel scientifique ou économique défini par le Comité ministériel, ou tout autre intérêt fondamental du pays défini par le Roi sur proposition du Comité ministériel »; l'article 8 précise ensuite ce qu'il faut entendre par " "activité qui menace ou pourrait menacer" : toute activité, individuelle ou collective, déployée à l'intérieur du pays ou à partir de l'étranger, qui peut avoir un rapport avec l'espionnage, l'ingérence, le terrorisme, l'extrémisme, la prolifération, les organisations sectaires nuisibles, les organisations criminelles; en ce compris la diffusion de propagande, l'encouragement ou le soutien direct ou indirect, notamment par la fourniture de moyens financiers, techniques ou logistiques, la livraison d'informations sur des objectifs potentiels, le développement des structures et du potentiel d'action et la réalisation des buts poursuivis »; enfin, l'alinéa 2 éclaircit le contenu du premier alinéa en présentant une série de définitions de certains de ses termes, et notamment le «terrorisme ». Loi organique des services de renseignement et de sécurité, M.B. 18 décembre 1998.

23. Voir par exemple sur ce sujet l'ouvrage collectif dirigé par Mazower M., The Policing of Politics in the Twentieth Century, Providence-Oxford, Berghahn Books (coll. « Historical Perspectives »), 1997.

24.. Calqué sur le Code napoléonien de 1810, le Code pénal belge est structuré en deux Livres : le Livre I établit les principes du droit pénal (droit pénal général), tandis que le Livre II est consacré à la définition des infractions (droit pénal spécial).

25.. Comme l'homicide volontaire ou les coups et blessures volontaires $\left(1^{\circ}\right)$, la prise d'otage $\left(2^{\circ}\right)$, la capture d'aéronef $\left(4^{\circ}\right)$, de navire $\left(5^{\circ}\right)$, les infractions liées à la fabrication et à l'emploi de produits explosifs $\left(7^{\circ}\right)$ ou à la mise au point d'armes bactériologiques $\left(10^{\circ}\right) 22$, par exemple.

26. Notamment la Ligue belge des droits de l'Homme et certaines organisations syndicales.

27.. Ainsi, le $\S 3,1^{\circ}$ mentionne « la destruction ou la dégradation massives, ou la provocation d'une inondation d'une infrastructure, d'un système de transport, d'une propriété publique ou privée, ayant pour effet de mettre en danger des vies humaines ou de produire des pertes économiques considérables, autres que celles visées au $\$ 2 »$.

28. " Constitue une infraction terroriste, l'infraction prévue aux $\S \S 2$ et 3 qui, de par sa nature ou son contexte, peut porter gravement atteinte à un pays ou à une organisation internationale et est commise intentionnellement dans le but d'intimider gravement une population ou de contraindre indûment des pouvoirs publics ou une organisation internationale à accomplir ou à s'abstenir d'accomplir un acte, ou de gravement déstabiliser ou détruire les structures fondamentales politiques, constitutionnelles, économiques ou sociales d'un pays ou d'une organisation internationale. » Art. 137, § $1^{\mathrm{er}}$ Code pénal.

29. Cour de cassation, section néerlandaise, $2^{\mathrm{e}}$ chambre, 11 septembre 2004.

30.. A. Braas (chev.), Précis de droit pénal, Bruxelles, Bruylant, 1946, p. 210.

31.. " Fait dont le caractère exclusif est de porter atteinte à la forme et à l'ordre politique d'une nation. » Cour de cassation, 12 mars 1855 ; « le propre des ces délits [politiques est] [...] de porter uniquement atteinte à l'ordre politique établi », Cour de cassation, 29 mars 1887.

32. A. Braas (chev.), op. cit., p. 211.

33.. Papadatos P., op. cit., p. 85.

34.. Foucault M., « Non au sexe roi » (entretien), M. Foucault, Dits et écrits, Paris, Gallimard (coll. « Quarto »), 2001, vol.II, p. 263. 
35.. Bigo D., « La mondialisation de l'(in)sécurité ? Réflexions sur le champ des professionnels de la gestion des inquiétudes et analytique de la transnationalisation des processus d'(in)sécurisation ", Cultures \& Conflits n58, été 2005, pp. 53-100.

36.. Ibid., p. 60.

37. Voir par exemple Garland D., The Culture of Control, Oxford, Oxford University Press, 2001.

38. (Nous soulignons) Ferri E., La Sociologie criminelle, cité par Papadatos P., op. cit., p. 46. 39.. Rappelons-le, l'élément intentionnel « terroriste » figurant dans la loi belge du 19 décembre 2003 est transcrit tel quel dans la décision-cadre du Conseil du 13 juin 2002.

40.. Ansart P., Idéologies, conflits et pouvoirs, Paris, PUF, 1977, p. 36.

41.. Garland D., The Culture of Control, op. cit., p. 12. Nous traduisons.

42.. .Kappeler V.E et A.E., " Speaking of Evil and Terrorism », M. Deflem (dir..), Terrorism and Counter-terrorism : criminological perspectives, Oxford, Elsevier, 2004, p. 183.

43. Dans le sens de la «méconnaissance-reconnaissance » de Bourdieu.

44.. Garland D., op. cit., p. 13. Nous traduisons.

45.. Voir Foucault M., Sécurité, Territoire, Population, Paris, Gallimard, 2004, pp. 362 et suivantes.

\section{RÉSUMÉS}

La loi du 19 décembre 2003 inscrivant au Code pénal des infractions terroristes spécifiques marque une nouvelle étape dans l'évolution du délit politique en Belgique. En effet, l'élément moral "politique» retenu dans la définition de ces infractions est conçu comme une circonstance aggravante, et contredit ainsi le principe du régime de faveur établi aux premières heures de la Belgique et progressivement restreint dans son application. Plus largement, cette « dé-politisation » du délit politique à travers le terrorisme renvoie à la gouvernementalité et au modèle démocratique contemporains, qui semblent induire une exclusion de l'idéologie hors du champ de ce qui constitue, selon Foucault, les « conditions du vrai et du faux ».

The $19^{\text {th }}$ December 2003 law inserting specific terrorist infractions in the penal Code sets a new step in the evolution of the political offence in Belgium: the "political » moral element in the definition of those infractions has indeed been conceived as an aggravating circumstance, opposing in that way the principle of the "régime de faveur " (preferential treatment) established by the newborn Belgium and progressively restricted in its application. At a larger scale, this "de-politization» of the political offence through terrorism can lead to contemporary governmentality and democratic model which seem to induce an exclusion of ideology out of the field of what Foucault calls « the conditions of true and false ».

\section{INDEX}

Mots-clés : délinquance, terrorisme

Index géographique : Benelux, Bruxelles, Europe 


\section{AUTEUR}

\section{MARTIN MOUCHERON}

Martin Moucheron est assistant de recherche à l'Unité de criminologie de l'Université catholique de Louvain. Criminologue et philosophe, il termine une thèse de doctorat en criminologie en tant qu'Aspirant du Fonds National de la Recherche Scientifique (FNRS) portant sur l'objet « terrorisme » dans la gouvernementalité en Belgique et en Europe. 